Harant, Jochen; Schiermeyer, Ingo:

A lower bound on the independence number of a graph in terms of degrees

URN: $\quad$ urn:nbn:de:gbv:ilm1-2020200134

\begin{tabular}{|c|c|}
\hline Original published in: & $\begin{array}{l}\text { Discussiones mathematicae. Graph theory / Uniwersytet Zielonogórski, } \\
\text { Wydział Matematyki, Informatyki i Ekonometrii. - Warsaw : De Gruyter } \\
\text { Open. - } 26 \text { (2006), 3, p. 431-437. }\end{array}$ \\
\hline Original published: & 2006 \\
\hline ISSN: & $2083-5892$ \\
\hline DOI: & 10.7151/dmgt.1335 \\
\hline \multirow[t]{2}{*}{ [Visited: } & 2020-01-17] \\
\hline & $\begin{array}{l}\text { This work is licensed under a Creative Commons Attribution- } \\
\text { NonCommercial-NoDerivatives } 3.0 \text { Unported license. } \\
\text { To view a copy of this license, visit } \\
\text { http://creativecommons.org/licenses/BY-NC-ND/3.0/ }\end{array}$ \\
\hline
\end{tabular}


Discussiones Mathematicae

Graph Theory 26 (2006) 431-437

\title{
A LOWER BOUND ON THE INDEPENDENCE NUMBER OF A GRAPH IN TERMS OF DEGREES
}

\author{
JOCHEN HARANT \\ Institut für Mathematik, TU Ilmenau \\ 98684 Ilmenau, Germany \\ AND \\ INGO SCHIERMEYER \\ Institut für Diskrete Mathematik und Algebra \\ TU Bergakademie Freiberg \\ 09596 Freiberg, Germany
}

\begin{abstract}
For a connected and non-complete graph, a new lower bound on its independence number is proved. It is shown that this bound is realizable by the well known efficient algorithm MIN.
\end{abstract}

Keywords: independence, stability, algorithm.

2000 Mathematics Subject Classification: 05C69, 05C85.

\section{Introduction And TheOrem}

Let $G$ be a finite, undirected, simple, non-complete, and connected graph on its vertex set $V(G)=\{1,2, \ldots, n\}$. For a subgraph $H$ of $G$ and for a vertex $i \in V(H)$ let $d_{H}(i)$ be the degree of $i$ in $H$, i.e., the cardinality of the neighbourhood $N_{H}(i) \subset V(H)$ of $i$ in $H$, and let $\delta(H)$ be the minimum degree of $H$. A subset $I$ of $V(G)$ is called independent if the subgraph of $G$ spanned by $I$ is edgeless. The independence number $\alpha(G)$ is the largest cardinality $|I|$ among all independent sets $I$ of $G$. The following algorithm MIN (cf. [8]) is a well known procedure to construct an independent set of a graph $G$. 
Algorithm MIN:

1. $G_{1}:=G, j:=1$

2. while $V\left(G_{j}\right) \neq \emptyset$ do

begin

choose $i_{j} \in V\left(G_{j}\right)$ with $d_{G_{j}}\left(i_{j}\right)=\delta\left(G_{j}\right)$, delete $\left\{i_{j}\right\} \cup N_{G_{j}}\left(i_{j}\right)$ to obtain $G_{j+1}$ and set $j:=j+1$;

end;

3. $k:=j-1$

STOP

Obviously, the set $\left\{i_{1}, i_{2}, \ldots, i_{k}\right\} \subset V(G)$ is an independent set of $G$ and therefore $\alpha(G) \geq k$ for every output $k$ of algorithm MIN. Let $k_{M I N}$ be the smallest $k$ Algorithm MIN provides for a fixed graph $G$. In the following Theorem a new lower bound on $k_{M I N}$ is established.

Theorem. Let $G$ be a finite, simple, connected, and non-complete graph on $n$ vertices with maximum degree $\Delta, n_{j}$ be the number of vertices of degree $j$ in $G$, and

$$
\begin{aligned}
x(j)= & \frac{j(j+1)}{j(j+1)-1}\left[\left(\frac{1}{j+1}-(\Delta-j)\right) n_{\Delta}+\left(\frac{1}{j+1}-(\Delta-j-1)\right) n_{\Delta-1}\right. \\
& \left.+\ldots+\left(\frac{1}{j+1}-1\right) n_{j+1}+\frac{n_{j}}{j+1}+\frac{n_{j-1}}{j}+\ldots+\frac{n_{1}}{2}-1\right]
\end{aligned}
$$

for $j \in\{\Delta, \Delta-1, \ldots, 1\}$.

(i) Then there is a unique $j_{0} \in\{\Delta, \Delta-1, \ldots, 1\}$ such that $0 \leq x\left(j_{0}\right)<$ $n_{\Delta}+\ldots+n_{j_{0}}$ and

$$
\begin{aligned}
k_{M I N} \geq & \left(\sum_{j=1}^{\Delta} \frac{n_{j}}{j+1}\right)+\frac{n_{\Delta}}{\Delta(\Delta+1)}+\frac{n_{\Delta}+n_{\Delta-1}}{(\Delta-1) \Delta} \\
& +\ldots+\frac{n_{\Delta}+\ldots+n_{j_{0}+1}}{\left(j_{0}+2\right)\left(j_{0}+1\right)}+\frac{x\left(j_{0}\right)}{\left(j_{0}+1\right) j_{0}} \\
= & 1+x\left(j_{0}\right)+n_{j_{0}+1}+2 n_{j_{0}+2}+\ldots+\left(\Delta-j_{0}\right) n_{\Delta} .
\end{aligned}
$$




\section{ProOF}

Let $d_{i}=d_{G}(i), i=1, \ldots, n$ and for $1 \leq k \leq d_{1}+\ldots+d_{n}+1$ let $f(k)=$ $\min \sum_{i=1}^{n} \frac{1}{d_{i}+1-x_{i}}$, where the minimum is taken over integers $x_{i}$ with $0 \leq$ $x_{i} \leq d_{i}$ and $\sum_{i=1}^{n} x_{i}=k-1$. Lemma 1 and Lemma 2 are proved in [7].

Lemma 1. $k_{M I N} \geq f\left(k_{M I N}\right)$.

Lemma 2. The following algorithm A calculates $f(k)$ :

Input: $F=\left\{d_{1}, d_{2}, \ldots, d_{n}\right\}, k \in\left\{1,2, \ldots, d_{1}+\ldots+d_{n}+1\right\}, j:=0$;

while $j<k-1$ do begin $F:=(F \backslash\{\max (F)\}) \cup\{\max (F)-1\} ; j:=j+1$ end. Output: $f(k)=\sum_{f \in F} \frac{1}{f+1}$.

Note that $F$ is a family, i.e., a member of $F$ may occur more than once. Given $k \in\left\{1,2, \ldots, d_{1}+\ldots+d_{n}+1\right\}$, in each of the $k-1$ steps of algorithm $A$ a maximum member $f$ of the current family $F$ is replaced by $f-1$.

If $k=d_{1}+\ldots+d_{n}+1$ then $f(k)=n$. If $1 \leq k \leq d_{1}+\ldots+d_{n}=n_{1}+2 n_{2}+$ $\ldots+\Delta n_{\Delta}$ then there are unique integers $j$ and $x$ with $j \in\{\Delta, \Delta-1, \ldots, 1\}$ and $0 \leq x<n_{\Delta}+\ldots+n_{j}$ such that $k-1=x+n_{j+1}+2 n_{j+2}+\ldots+(\Delta-j) n_{\Delta}=$ $n_{\Delta}+\left(n_{\Delta}+n_{\Delta-1}\right)+\ldots+\left(n_{\Delta}+n_{\Delta-1}+\ldots+n_{j+1}\right)+x$. With this expression for $k-1$ the part cut away by algorithm $A$ is illustrated in Figure 1.

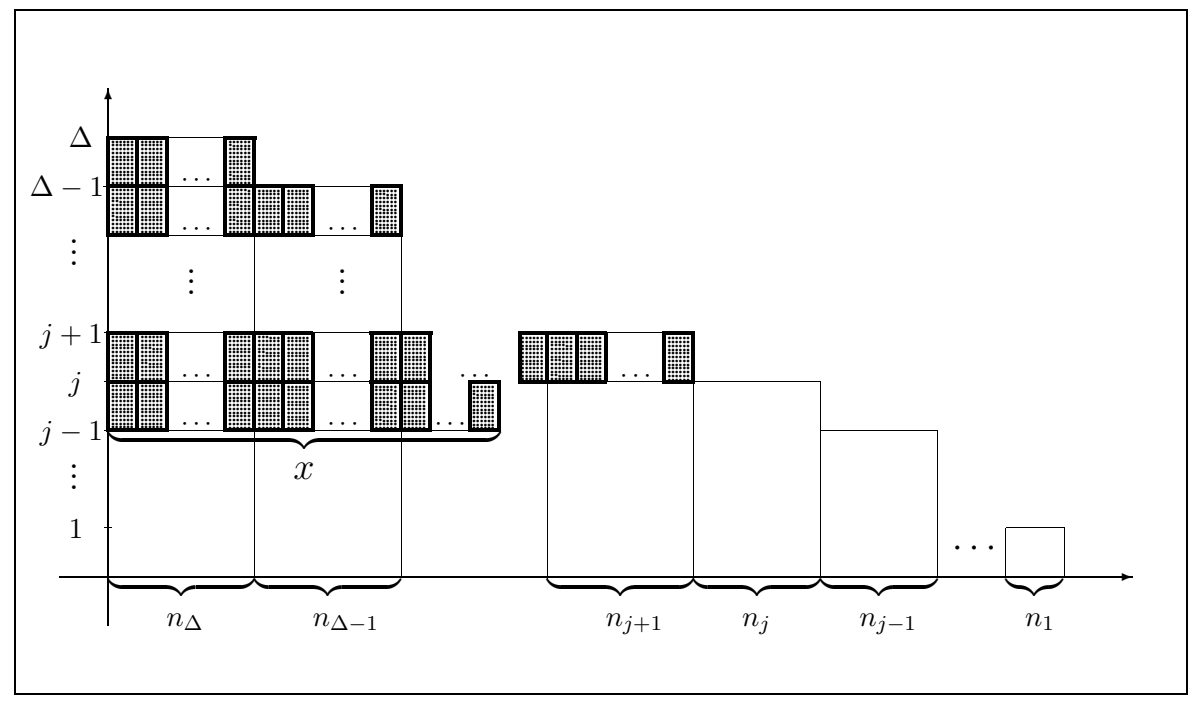

Figure 1 
Hence, after applying algorithm $A$, the family $F$ contains the member $j-1$ exactly $x+n_{j-1}$ times, the member $j$ exactly $n_{\Delta}+\ldots+n_{j}-x$ times, and all other members of $F$ being smaller than $j-1$ at the beginning remain unchanched. Thus, the following Lemma 3 is proved.

\section{Lemma 3.}

(i) Given $k \in\left\{1, \ldots, d_{1}+\ldots+d_{n}\right\}$, there are unique integers $j$ and $x$ with $j \in\{\Delta, \Delta-1, \ldots, 1\}$ and $x \in\left\{0, \ldots, n_{\Delta}+\ldots+n_{j}-1\right\}$ such that

$$
\begin{aligned}
k-1 & =n_{\Delta}+\left(n_{\Delta}+n_{\Delta-1}\right)+\ldots+\left(n_{\Delta}+n_{\Delta-1}+\ldots+n_{j+1}\right)+x \\
& =x+n_{j+1}+2 n_{j+2}+\ldots+(\Delta-j) n_{\Delta}
\end{aligned}
$$

and

(ii) $f(k)=\left(n_{\Delta}+\ldots+n_{j}-x\right) \frac{1}{j+1}+\frac{x}{j}+\frac{n_{j-1}}{j}+\ldots+\frac{n_{1}}{2}$

$$
=\left(n_{\Delta}+\ldots+n_{j}\right) \frac{1}{j+1}+\frac{x}{j(j+1)}+\frac{n_{j-1}}{j}+\ldots+\frac{n_{1}}{2} \text { for that } k .
$$

Lemma 4. If $k=1+x+n_{j+1}+2 n_{j+2}+\ldots+(\Delta-j) n_{\Delta}$ with $j \in$ $\{\Delta, \Delta-1, \ldots, 1\}$ and $x \in\left\{0, \ldots, n_{\Delta}+\ldots+n_{j}-1\right\}$, then $f(k+1)-f(k)=$ $\frac{1}{j(j+1)}$.

Proof of Lemma 4. If $x \leq n_{\Delta}+\ldots+n_{j}-2$ then $k+1=1+$ $(x+1)+n_{j+1}+2 n_{j+2}+\ldots+(\Delta-j) n_{\Delta}$ and if $x=n_{\Delta}+\ldots+n_{j}-1$ then $k+1=1+n_{j}+2 n_{j+1}+\ldots+(\Delta-j+1) n_{\Delta}$. In both cases Lemma 3 implies Lemma 4.

Using Lemma 3, the calculation of $f(k)$ is possible now without taking a minimum and without using algorithm $\mathrm{A}$. In the sequel, we will define the function $f$ for real $k \in\left[1, d_{1}+\ldots+d_{n}+1\right)$ and show that the function $g(k)=k-f(k)$ is continuous and strictly increasing on $\left[1, d_{1}+\ldots+d_{n}+1\right)$. Finally, using $g(1)<0$ and $g\left(k_{M I N}\right) \geq 0$, the lower bound $k_{0}$ on $k_{M I N}$ is the unique solution of the equation $k=f(k)$.

Thus, for given integer $j \in\{\Delta, \Delta-1, \ldots, 1\}$ and real number $x$ with $0 \leq x<n_{\Delta}+\ldots+n_{j}$ let the real numbers $k$ and $f(k)$ (implicitely) be defined as $k=1+x+n_{j+1}+2 n_{j+2}+\ldots+(\Delta-j) n_{\Delta}$ and $f(k)=$ $\left(n_{\Delta}+\ldots+n_{j}\right) \frac{1}{j+1}+\frac{x}{j(j+1)}+\frac{n_{j-1}}{j}+\ldots+\frac{n_{1}}{2}$. 
Lemma 5. The function $g$ with $g(k)=k-f(k)$ is continuous and strictly increasing on $\left[1, d_{1}+\ldots+d_{n}+1\right)$.

Proof of Lemma 5. First, let $j \in\{\Delta, \Delta-1, \ldots, 1\}$ be fixed. Then $k=1+x+n_{j+1}+2 n_{j+2}+\ldots+(\Delta-j) n_{\Delta}$ with $0 \leq x<n_{\Delta}+\ldots+n_{j}$ belongs to the interval $I(j)=\left[1+n_{j+1}+2 n_{j+2}+\ldots+(\Delta-j) n_{\Delta}, 1+n_{j}+\right.$ $\left.2 n_{j+1}+\ldots+(\Delta-j+1) n_{\Delta}\right)$. Obviously $g$ is continuous on $I(j)$ and, because $g(k+\epsilon)-g(k)=\epsilon-\frac{\epsilon}{j(j+1)}$ and $j(j+1) \geq 2, g$ is strictly increasing on $I(j)$.

Now consider $g$ on $\left[1, \ldots, d_{1}+\ldots+d_{n}+1\right)$ and note that $I(\Delta) \cup \ldots \cup I(1)=$ $\left[1, \ldots, d_{1}+\ldots+d_{n}+1\right)$ and $I(j) \cap I\left(j^{\prime}\right)=\emptyset$ if $j \neq j^{\prime}$. It is easy to see that $g$ is also continuous in $k=1+n_{j+1}+2 n_{j+2}+\ldots+(\Delta-j) n_{\Delta}$ for $j \in\{\Delta-1, \Delta-2, \ldots, 2\}$ and we are done.

In $[2,12]$ the well known Caro-Wei-bound $C W=\sum_{j=1}^{\Delta} \frac{n_{j}}{j+1}$ is proved to be a lower bound on $\alpha(G)$ and being tight if and only if $G$ is complete. With our assumption that $G$ is non-complete, $g(1)=1-\sum_{j=1}^{\Delta} \frac{n_{j}}{j+1}<0$ and $g\left(k_{M I N}\right) \geq 0$ by Lemma 1 . As a consequence of Lemma 5 there is a unique zero $k_{0}=1+x\left(j_{0}\right)+n_{j_{0}+1}+2 n_{j_{0}+2}+\ldots+\left(\Delta-j_{0}\right) n_{\Delta}$ of $g$ with $1<k_{0} \leq k_{M I N}$ and $0 \leq x\left(j_{0}\right)<n_{\Delta}+\ldots+n_{j_{0}}$. Considering the equation $f(k)=k$ we obtain

Lemma 6. If $j \in\{\Delta, \Delta-1, \ldots, 1\}$ and $k=1+x+n_{j+1}+2 n_{j+2}+\ldots+$ $(\Delta-j) n_{\Delta}$ with $0 \leq x<n_{\Delta}+\ldots+n_{j}$, then $f(k)=k$ if and only if

$$
\begin{aligned}
x= & \frac{j(j+1)}{j(j+1)-1}\left[\left(\frac{1}{j+1}-(\Delta-j)\right) n_{\Delta}\right. \\
& \left.+\ldots+\left(\frac{1}{j+1}-1\right) n_{j+1}+\frac{n_{j}}{j+1}+\ldots+\frac{n_{1}}{2}-1\right] .
\end{aligned}
$$

Now we complete the proof of the Theorem. Assume there is $j_{1} \in\{\Delta, \Delta-$ $1, \ldots, 1\}$ with $j_{1} \neq j_{0}, x=\frac{j_{1}\left(j_{1}+1\right)}{j_{1}\left(j_{1}+1\right)-1}\left[\left(\frac{1}{j_{1}+1}-\left(\Delta-j_{1}\right)\right) n_{\Delta}+\ldots+\left(\frac{1}{j_{1}+1}-1\right)\right.$ $\left.n_{j_{1}+1}+\frac{n_{j_{1}}}{j_{1}+1}+\ldots+\frac{n_{1}}{2}-1\right]$, and $0 \leq x<n_{\Delta}+\ldots+n_{j_{1}}$. Then $k_{1}=$ $1+x\left(j_{1}\right)+n_{j_{1}+1}+2 n_{j_{1}+2}+\ldots+\left(\Delta-j_{1}\right) n_{\Delta}$ is a solution of the equation $f(k)=k$ by Lemma 6 and $k_{0} \neq k_{1}$ by Lemma 3 (i) contradicting the uniqueness of $k_{0}$.

With $k_{0}=f\left(k_{0}\right)=f(1)+(f(2)-f(1))+\ldots+\left(f\left(\left\lfloor k_{0}\right\rfloor\right)-f\left(\left\lfloor k_{0}\right\rfloor-1\right)\right)+$ $\left(f\left(k_{0}\right)-f\left(\left\lfloor k_{0}\right\rfloor\right)\right)$ and Lemma 4 we have $f\left(k_{0}\right)=\left(\sum_{j=1}^{\Delta} \frac{n_{j}}{j+1}\right)+\frac{n_{\Delta}}{\Delta(\Delta+1)}+$ $\frac{n_{\Delta}+n_{\Delta-1}}{(\Delta-1) \Delta}+\ldots+\frac{n_{\Delta}+\ldots+n_{j_{0}+1}}{\left(j_{0}+2\right)\left(j_{0}+1\right)}+\frac{x\left(j_{0}\right)}{\left(j_{0}+1\right) j_{0}}$ and the Theorem is proved. 
Many lower bounds on $\alpha(G)$ are known (cf. [1, 2, 3, 4, 5, 6, 8, 9, 10, 11]). If we compare them with $k_{0}$, let us remark here that, by the Theorem,

$$
\begin{aligned}
& k_{0}=C W+\frac{n_{\Delta}}{\Delta(\Delta+1)}+\frac{n_{\Delta}+n_{\Delta-1}}{(\Delta-1) \Delta}+\ldots+\frac{n_{\Delta}+\ldots+n_{j_{0}+1}}{\left(j_{0}+2\right)\left(j_{0}+1\right)}+\frac{x\left(j_{0}\right)}{\left(j_{0}+1\right) j_{0}}
\end{aligned}
$$

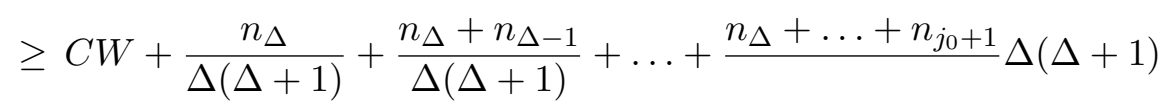

$$
\begin{aligned}
& +\frac{x\left(j_{0}\right)}{\Delta(\Delta+1)}=C W+\frac{k_{0}-1}{\Delta(\Delta+1)} \text {. }
\end{aligned}
$$

This implies $k_{0} \geq C W+\frac{C W-1}{\Delta(\Delta+1)-1}$ improving the well known lower bound $C W+\frac{C W-1}{\Delta(\Delta+1)}$ on $\alpha(G)$ by O. Murphy ([8]).

In [6] it was established $\alpha \geq \frac{C W^{2}}{C W-\sum_{i j \in E(G)}\left(d_{i}-d_{j}\right)^{2} q_{i}^{2} q_{j}^{2}}$, and S.M. Selkow ([9]) proved $\alpha \geq \sum_{i=1}^{n} q_{i}\left(1+\max \left\{0, d_{i} q_{i}-\sum_{i j \in E(G)} q_{j}\right\}\right)$, where $q_{i}=\frac{1}{d_{i}+1}$ and $E(G)$ is the edge set of $G$. Both bounds equal $C W$ if the graph is regular, however, Murphy's bound and therefore also $k_{0}$ are considerably larger in that case. For a star $K_{1, p}$ on $p+1$ vertices we have the converse situation, i.e., $k_{0}$ is not comparable with these bounds in $[6,9]$.

\section{Acknowledgement}

The authors want to thank Olga Gross (Ilmenau) and Martin Sonntag (Freiberg) for their hints and remarks.

\section{REFERENCES}

[1] E. Bertram and P. Horak, Lower bounds on the independence number, Geombinatorics V (1996) 93-98.

[2] Y. Caro, New results on the independence number (Technical Report. Tel-Aviv University, 1979).

[3] Y. Caro and Z. Tuza, Improved lower bounds on k-independence, J. Graph Theory 15 (1991) 99-107.

[4] S. Fajtlowicz, On the size of independent sets in graphs, Proc. 9th S-E Conf. on Combinatorics, Graph Theory and Computing, Boca Raton 1978, 269-274.

[5] S. Fajtlowicz, Independence, clique size and maximum degree, Combinatorica 4 (1984) 35-38. 
[6] J. Harant, A lower bound on the independence number of a graph, Discrete Math. 188 (1998) 239-243.

[7] J. Harant and I. Schiermeyer, On the independence number of a graph in terms of order and size, Discrete Math. 232 (2001) 131-138.

[8] O. Murphy, Lower bounds on the stability number of graphs computed in terms of degrees, Discrete Math. 90 (1991) 207-211.

[9] S.M. Selkow, The independence number of a graph in terms of degrees, Discrete Math. 132 (1994) 363-365.

[10] J.B. Shearer, A note on the independence number of triangle-free graphs, Discrete Math. 46 (1983) 83-87.

[11] J.B. Shearer, A note on the independence number of triangle-free graphs, II, J. Combin. Theory (B) 53 (1991) 300-307.

[12] V.K. Wei, A lower bound on the stability number of a simple graph (Bell Laboratories Technical Memorandum 81-11217-9, Murray Hill, NJ, 1981).

Received 28 November 2005

Revised 28 June 2006 